\section{Diagnosis using methylation}

Early diagnosis enables substantially better outcomes among patients with colorectal cancer (CRC). Several methods of screening for early stage CRC exist, although these are either invasive (colonoscopy) or lack sufficient sensitivity (serum carcinoembryonic antigen (CEA)). Now, data from a large-cohort study reveal the potential of methylation of cell-free DNA (cfDNA) in the detection of CRC.

Researchers compared methylation data from 459 CRC samples analysed as part of The Cancer Genome Atlas with methylation profiles from 754 individuals without CRC.

The diagnostic performance of classification based on methylation profile was investigated in the training and validation sets (comprising 1,202 and 620 patients, respectively), and revealed sensitivities and specificities for CRC detection of $87.5 \%$ and $89.9 \%$ in the training set, and $87.9 \%$ and $89.6 \%$ in the validation set, respectively. Comparisons of methylation-based CRC detection with CEA testing revealed the superiority of the methylation-based approach (AUC 0.96 versus 0.67 ). A composite (cd)-score, based on nine different methylation markers, was then constructed.

cd-score was then combined with other prognostic features to develop a combined prognostic (cp) score. Application of cp-score revealed significant differences in overall survival durations between patients with CRCs classified as high-risk versus low-risk in the training set $(P<0.001)$ and validation set $(P=0.0012)$ : cd-score was independently predictive of survival in both sets.

Owing to the challenges associated with implementation of complex biomarkers, researchers selected the most promising single marker (methylation of site CpG cg10673833) for prospective

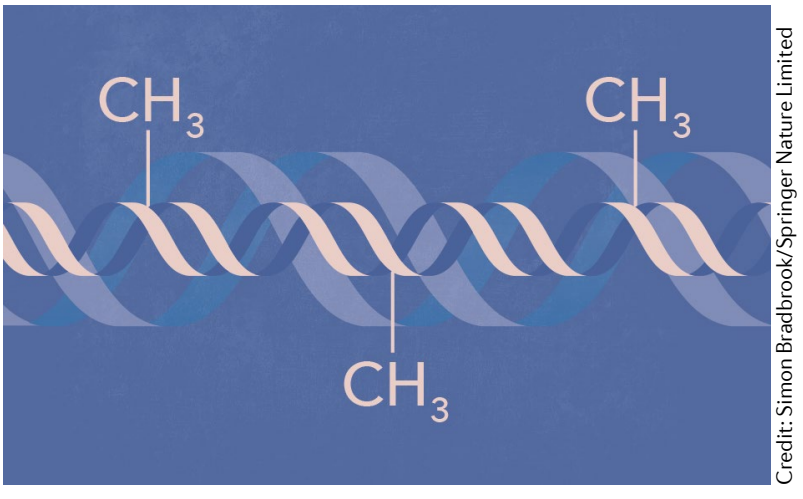

testing. A total of 19 of 21 CRCs were identified using this test (sensitivity $89.7 \%$, specificity $86.8 \%$ ) in a cohort of 1,493 individuals with a high risk of CRC undergoing colonoscopy.

Lead author Rui-Hua Xu summarizes: "Our most important finding is that accurate early diagnosis of CRC is feasible by accurate early diagnosis of $\mathrm{CRC}$ is feasible by detecting cfDNA methylation markers detecting cfDNA methylation markers in just a few mls of blood". When asked about future directions, $\mathrm{Xu}$ adds: "we need to design a randomized, population-based study to compare this non-invasive screening strategy with other tests".

Peter Sidaway

ORIGINAL ARTICLE Luo, H. et al. Sci. Transl Med.

524, eaax7533 (2020)

\title{
Moving pembrolizumab forwards
}

Immune-checkpoint inhibitors (ICls) have the potential to induce long-term remission of cancer, but have disappointing efficacy in patients with breast cancer, at least as monotherapy for advanced-stage disease. Now, new data from two trials indicate that ICls might be more effective if combined with neoadjuvant chemotherapy.

As part of the adaptively randomized phase II I-SPY2 trial, 40 women with high-risk HER2-negative and hormone receptor-positive disease (HER2 ${ }^{-} / \mathrm{HR}^{+}$) and 29 with triple-negative breast cancer (TNBC) received pembrolizumab added to neoadjuvant chemotherapy (paclitaxel then doxorubicin and cyclophosphamide). The pathological complete response (pCR) rates in the HER2- population overall, the $\mathrm{HER}^{-} / \mathrm{HR}^{+}$subgroup and the TNBC subgroup were more than double those observed in a total of 181 women treated with chemotherapy alone:

$44 \%$ versus $17 \%, 30 \%$ versus $13 \%$ and $60 \%$ versus $22 \%$, respectively. In all three

The pathological complete response ( $\mathrm{pCR}$ ) rates ... were more than double those observed ... with chemotherapy alone

5
settings, the probability of success in a 300-patient, subtype-specific phase III trial of the pembrolizumab combination was $>85 \%$. In an immature exploratory analysis of event-free survival (EFS), patients with a $\mathrm{pCR}$ faired very well, regardless of which treatment they received (hazard ratios $<0.4$ relative to their counterparts without a pCR).

Immune-related adverse events, particularly thyroid and adrenal endocrinopathies (frequency of 15.9\% and $8.7 \%$, respectively), occurred with pembrolizumab, but not with chemotherapy alone. Thus, identifying predictive biomarkers will be important to avoid potentially unnecessary and toxic treatment of patients who often have excellent outcomes with current therapies.

In the ongoing phase III KEYNOTE-522 trial, patients with TNBC are being randomly assigned $(2: 1)$ to receive neoadjuvant chemotherapy (paclitaxel and carboplatin then cyclophosphamide plus either epirubicin or doxorubicin) plus either pembrolizumab or placebo, with stratification by PD-L1 status. In an interim analysis of data from 602 patients, the $\mathrm{pCR}$ rates were $64.8 \%$ with the $\mathrm{ICl}$ versus $51.2 \%$ with placebo $(P<0.001)$; the rates were $68.9 \%$ versus $54.9 \%$ and $45.3 \%$ versus $30.3 \%$ in the PD-L1 ${ }^{+}$and PD-L1 ${ }^{-}$subgroups, respectively, suggesting a similar degree of benefit ( 15\%). At a median follow-up duration of 15.5 months, pembrolizumab was also associated with a trend towards improved EFS (hazard ratio $0.63,95 \% \mathrm{Cl}$ $0.43-0.93)$. The toxicity profiles were as expected for each treatment, with similar rates of grade $\geq 3$ treatment-related adverse events (78\% versus $73 \%$ ).

These promising results warrant further evaluation of the potential long-term survival benefits.

David Killock

ORIGINAL ARTICLES Nanda, R. et al. JAMA Oncol. https://doi.org/10.1001/jamaoncol.2019.6650 (2020)|Schmid, P. et al. N. Engl.J. Med. 382, 810-821 (2020) 